JOURNAL OF MODERN OPTICS, 2001, VOL. 48, NO. 5, $811-829$

\title{
Refractive analysis of the human cornea through propagated fields
}

\author{
CARLOS ILLUECA ${ }^{1}$, DAVID MAS ${ }^{1}$, JORGE PEREZ ${ }^{1}$, \\ ALVARO M. PONS ${ }^{2}$ and JOSE M. ARTIGAS ${ }^{2}$ \\ 1 Dept. Interuniversitari d'Òptica,Universitat d'Alacant, P.O. Box 99, \\ E - 03080, Alacant, Spain \\ 2 Dept. Interuniversitari d'Òptica, Universitat de València, C/ Doctor \\ Moliner 50, 46100 Burjassot, Valencia, Spain
}

(Received 31 May 2000)

\begin{abstract}
A new technique for analysing the optical quality of the human cornea is presented. Corneal maps are obtained through keratographies and then converted into phase maps. The propagated fields generated from this surface are plotted and studied. It is shown that any irregularity in the cornea affects the propagated field and the energy distribution at the focal plane. Simple applications are also indicated.
\end{abstract}

\section{Introduction}

Modelling the eye requires full access to all parts of it. Visual tests that are commonly used in optometry provide the overall response of the eye, from a subjective point of view. These tests are enough to correct regular refractive ametropias and for qualitative diagnosis of more complicated problems. Unfortunately they cannot provide quantitative information of the different media separately, and thus other non-invasive objective techniques must be found.

Different objective methods have been applied to measure the optical impulse response of the eye (excluding the retinal response) [1, 2]. The double-pass technique has been successfully applied to obtain the modulation transfer function of the optics of the visual system [3], but, again, the different surfaces are not directly accessible.

Among all the different parts of the eye, the most important is the cornea. This surface is the interface between the air and the inner media inside the ocular globe, and thus it is responsible for the major part of the refractive power of the eye. As a consequence of this, it must have an important influence on the final image quality [4]. The optical quality of the cornea can be studied through its geometry, so that methods to obtain its curvature at any point are necessary. This is achieved by use of videokeratographs [5].

Topographic maps of the cornea are in common use in ophtalmological clinics. These maps allow modelling the cornea as a refractive surface. This information can be combined with the results obtained through the double-pass technique to obtain the response of the inner parts of the eye. 
Analysis of the corneal surface has been usually done through Zernike polynomials [6], which are in common use in the study of aberrations in lenses [7]. Zernike polynomials allow one to separate and classify the different irregularities that affect the refractive surface. Among others, the main advantage of these polynomials is that adequate choice of the basis permits description of any order aberrations, including Seidel aberrations. Therefore, evolution of any particular aberration can be easily analysed. Zernike polynomials allow one to also obtain the modulation transfer function of the cornea, and how this is affected by the different irregularities.

In some cases, Zernike decomposition, may be a too powerful tool. In some cases, the form of the surface itself is not as important as the propagation patterns that it generates. One may be interested in the light distribution at an image point, or how a test image propagates after passing through the cornea. For all these purposes, the Zernike description of the surface may be useful, but not absolutely necessary, and may be wasteful of computation time.

In this paper we propose a new method to evaluate the optical performance of a cornea through the study of the diffraction patterns it generates. Cornea data are directly extracted using videokeratography. The image obtained is processed to get a clean surface image [8] which acts as a standard dioptric surface. The convergence of the rays after the cornea and the light distribution around the focus plane is useful for the study of the different ametropias and any other deformations in the cornea surface.

Videokeratographs are intended purely for qualitative measurements, thus the data obtained with this technology are limited. The study that follows is not intended to be an exhaustive analysis of corneal aberrations. The objective here is to present the main advantages of a novel technique from a qualitative point of view. We are not interested in regular ametropias of the eye (myopia, hyperopia and astigmatism), since they can easily be detected and corrected by simple methods. Thus, little attention will be given to these problems although their presence will be noted. In our particular case, we are more interested in corneal diseases such as keratoconus and leukomas. With the method developed here, the presence of these problems can be clearly established.

The novelty and impact of the method that will be introduced is clear, since it allows an objective study in vivo of the optical quality of a cornea, independently of the rest of the eye. This new technique does not substitute any of the existing methods. It may be considered as a complementary method, which can be combined with the others to obtain better knowledge of the optical performance of the human eye.

In sections 2 and 3, an algorithm for the calculation of propagation patterns is introduced: the first section deals with the relation between the fractional Fourier transform and the Fresnel integral. In the second, this relation is exploited to design a fast algorithm for numerical evaluation of this integral. In section 4 , the use of this algorithm for studying light propagation after passing through a lens is detailed.

In section 5, the fundamentals of videokeratography are described. The methodology is briefly explained and the main equations are deduced. In section 6 the analyses of several cornea are discussed. In the final section, the main conclusions are outlined. 


\section{Fresnel patterns calculation through the fractional Fourier transform}

Since the fractional Fourier transform (FRT) was introduced to the optics community in 1993 [9, 10] a large number of publications have successfully developed this operation in the area of signal processing and have extended the concept of 'fractionalization' to other mathematical transformations in optics (see [11] for an extensive review of these operations and their applications).

FRT is a generalization of the ordinary Fourier transform depending on a parameter $p$ ( $p=1$ for the ordinary Fourier transform) and can be interpreted as a rotation in a space-frequency domain by an angle $p_{\pi / 2}$ of the original signal distribution. From a physical point of view, the FRT describes the diffraction patterns of a light distribution when it propagates through a GRIN optical fibre. From this perspective since both the FRT and the Fresnel integral describe light propagation, it is clear that they must be related [12]. In [13], a simple connection between the FRT and Fresnel propagation was established. This relation was developed later in [14] and [15], and it allows the possible application of the FRT transformation to many optical propagation problems.

In our particular case, a Fresnel through FR'T algorithm was proposed for the study of light propagation inside a Gullstrand-Emsley theoretical eye [16]. This technique allowed the obtention of a retinal image and thus derivation of the modulation transfer function of the eye.

The Fresnel pattern produced by an object $u_{0}$ at a distance $z$ when it is illuminated by a monochromatic plane wave with wavelength $\lambda$, can be expressed as:

$$
u_{z}\left(x_{z}\right)=\exp \left(\mathrm{i} \frac{\pi}{\lambda z} x_{z}^{2}\right) \int_{-\infty}^{+\infty}\left[u_{0}\left(x_{0}\right) \exp \left(\mathrm{i} \frac{\pi}{\lambda z} x_{0}^{2}\right)\right] \exp \left(-\mathrm{i} \frac{2 \pi}{\lambda z} x_{z} x_{0}\right) \mathrm{d} x_{0}
$$

where constant factors have been dropped. In this expression and in the remainder of this manuscript, we use a 1-D formulation. Extension to 2D is straightforward.

As stated in the previous section, we propose here a method for the analysis of the optical quality of the cornea. This method consists of defining the topography of this surface and studying the diffraction field generated by it. This requires an accurate numerical evaluation of expression (1).

In [15], several topics regarding the numerical calculation of Fresnel patterns are discussed. There, it is shown that, independently of the evaluation method, it is not possible to obtain good sampling conditions valid for the whole range of distances from zero to infinity and thus, a compromise result has to be used.

Among other fast Fourier transform (FFT) based methods that are presented in [15], here we choose one that permits evaluation of a Fresnel pattern through an equivalent FRT pattern. This method provides good evaluation of the amplitude of the diffraction integral for every distance considered. Let us recall that our goal is to check the optical quality of a human cornea in order to obtain better visual performance. Thus, we are not interested in the phase information of the light which is lost at the end of the vision process.

The fractional Fourier transform (FRT) of order $0<p<2$ of an input function $u_{0}\left(x_{0}\right)$ provided by a Lohmann Type II system [10] (see figure 1) can be expressed as: 

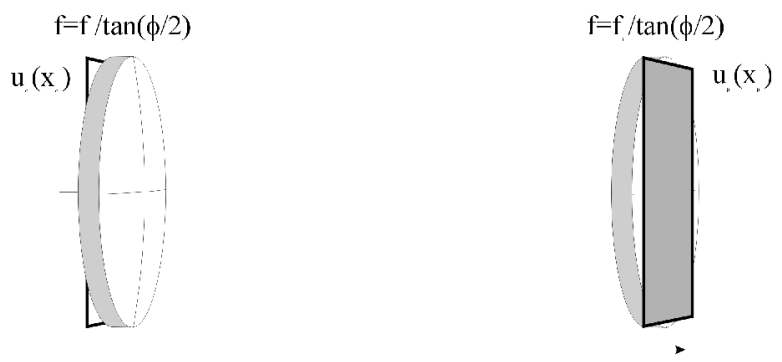

$$
z^{\prime}=f \sin (\phi)
$$

Figure 1. Lohmann's type II optical setup for obtaining an FR'T distribution of order $p=2 \phi / \pi$.

$$
\begin{aligned}
u_{p}\left(x_{p}\right)= & \exp \left(\frac{\mathrm{i}_{\pi}}{\lambda f_{1} \tan \phi} x_{p}^{2}\right) \int_{-\infty}^{\infty} u\left(x_{0}\right) \exp \left(\frac{\mathrm{i}_{\pi}}{\lambda f_{1} \tan \phi} x_{0}^{2}\right) \\
& \times \exp \left(-\frac{\mathrm{i} 2_{\pi}}{\lambda f_{1} \sin \phi} x_{0} x_{p}\right) \mathrm{d} x_{0},
\end{aligned}
$$

where $\phi=p_{\pi} / 2, \lambda$ the illuminating-light wavelength and $f_{1}$ an arbitrary fixed length. In general, the product $\lambda f_{1}$ will act as a controllable scaling factor. When this expression is compared with the Fresnel integral, for a distance $z=f_{1} \tan \phi$, the following relation is obtained $[12,15]$ :

$$
\begin{aligned}
u_{z}\left(\frac{x_{p}}{\beta}\right) & =\exp \left[\frac{\mathrm{i}_{\pi}}{\lambda f_{1}} \frac{x_{p}^{2}}{\sin \phi \cos \phi}\right] \int_{-\infty}^{\infty} u_{0}\left(x_{0}\right) \exp \left(\frac{\mathrm{i}_{\pi}}{\lambda f_{1} \tan \phi} x_{0}^{2}\right) \\
& \times \exp \left(-\frac{\mathrm{i} 2 \pi}{\lambda f_{1} \sin \phi} x_{0} x_{p}\right) \mathrm{d} x_{0} \\
& =\exp \left(\mathrm{i}_{\pi} x_{p}^{2} \frac{\tan \phi}{\lambda f_{1}}\right) F^{p}\left[u_{0}\left(x_{0}\right)\right] .
\end{aligned}
$$

Note that, aside of a scale and a global phase factor, the FRT and the diffraction integral coincide.

Since we are interested only in amplitude diffraction patterns, we can drop the exponential factor in expression (3) and full parallelism between the FRT and the diffraction integral can be established. Thus, we will use the fast-FRT calculation algorithm as a fast-Fresnel calculation algorithm, without further considerations (see [15] for more details).

\section{Fast FRT calculation algorithm}

Two different algorithms for FRT calculation through the FFT are described in $[17,18]$. Although both of them may provide good results for FRT pattern calculations, the method described in the first reference (fast-FRT method) provides better results than the other one when it is converted into a Fresnelcalculation algorithm [15]. Thus, in this communication, this algorithm will be used. 
The fast-FRT method consists of breaking the FRT integral into different cascaded processes. The algorithm is based on Lohmann's Type II configuration (see figure 1). In this method, the different elements affecting the light distribution, i.e. lens, free-space propagation and a second lens, are numerically evaluated and their effect applied to the input distribution. Free space propagation is accomplished, by convenience, in the Fourier domain [17].

The final discrete expression for the FRT obtained in this way is

$$
\begin{aligned}
& \left(U_{p}\right)_{\mu}=\exp \left[\mathrm{i} \frac{-\pi \operatorname{sgn}[\sin \phi]+2 \phi+\pi}{4}\right] \exp \left[-\mathrm{i} \frac{\pi \mu^{2}}{N} \tan (\phi / 2)\right] \times D F T^{-1}\{\cdots\}, \\
& \{\cdots\}=\left\{\exp \left[-\mathrm{i} \frac{\pi^{m^{2}}}{N} \sin \phi\right] \times D F T\left\{u_{0}\left(\frac{m \Delta^{x_{0}}}{N}\right) \exp \left[-\mathrm{i} \frac{\pi^{m^{2}}}{N} \tan (\phi / 2)\right]\right\}\right\},
\end{aligned}
$$

where the relations that have to be fulfilled between input and output sampling areas, derived from Discrete Fourier Transform [19] theory are:

$$
\Delta^{x_{0}} \Delta^{x_{p}}=\lambda f_{1} N
$$

where the subindex $p$ refers to the fractional domain. From the free space propagation in the Fourier domain, we obtain [17]:

$$
\Delta^{x_{0}}=\Delta^{x_{p}}=\sqrt{\lambda_{1} N} \text {. }
$$

We will assume that conditions for a fair sampling of expression (4) are obtained by application of the Nyquist theorem only on the quadratic phase factors affecting the signal. These conditions are:

$$
\text { (a) }|\sin (\phi)| \leq 1 \quad \text { (b) } \quad|\tan (\phi / 2)| \leq 1 .
$$

Condition (7a) is always fulfilled, while $(7 b)$ holds only for $\phi \leq \pi / 2$, or equivalently, $p \leq 1$. This range covers all domains between object and Fourier domain, and convenient application of the properties of the FRT will permit the extension of the algorithm to any arbitrary range [9].

Let us recall here that the method described in equation (3) will provide a scaled version of a Fresnel distribution. This scale is inherent to the process and, in fact helps the accuracy of the algorithm, since it impedes the divergence of the field for large $z$ values. The real size of the diffraction pattern obtained through the FRT method must be calculated a posteriori, and is

$$
\Delta^{x_{z}}=\frac{\Delta^{x_{p}}}{\beta}=\frac{\lambda^{N}}{\Delta^{x_{0}}} \sqrt{z^{2}+f_{1}^{2}}
$$

\section{Lenses and propagated fields}

Let us consider expression (3):

$$
u_{z}\left(\frac{x_{p}}{\beta}\right)=\exp \left(\mathrm{i}_{\pi} x_{p}^{2} \frac{\tan \phi}{\lambda f_{1}}\right) F^{p}\left[u_{0}\left(x_{0}\right)\right]
$$

and take a phase-only object. In our case, we will choose a single lens. It is clear that if we calculate the propagation of light after passing through the lens, we will obtain a field converging towards the geometrical focus. This is exactly what is 


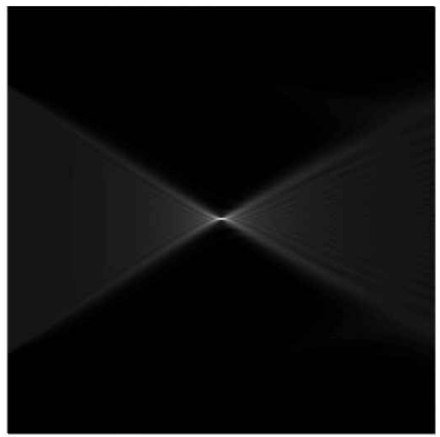

(a)
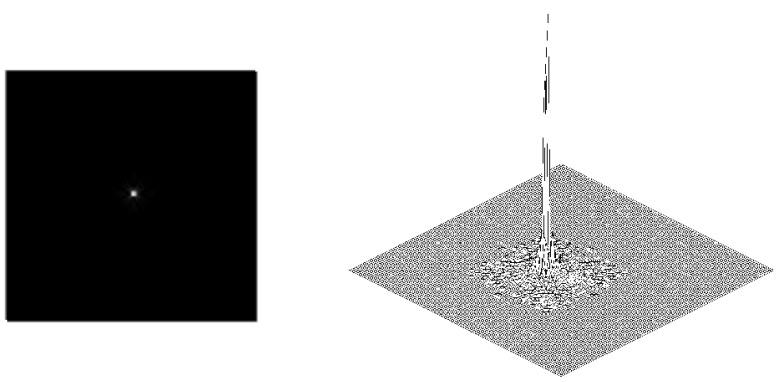

(b)

Figure 2. Spherical lens: (a) Propagation field generated by an arbitrary meridian. (b) Light distribution at the focus plane.

represented in figure 2(a). There we plot the field from the lens to infinity (leftright direction). Note that the horizontal scale does not represent distance itself but the order of the associated FRT. Bearing in mind the relation between the fractional order and the distance, what is finally represented in the horizontal axis is:

$$
z=f_{1} \tan \left(p_{\pi / 2}\right) \Longrightarrow p=\frac{2}{\pi} \tan ^{-1}\left(\frac{z}{f_{1}}\right) .
$$

In figure $2(b)$ we present the distribution of the energy at the geometrical focus of the lens. Note that border and sampling effects have little effect on the quality of the focus spot. For this example, we have chosen a lens of $8 \mathrm{~mm}$ diameter, and a refractive power of 50 diopters, in order to approximate our results to those we will present later with the cornea surface as a lens.

In figure 3 we present the optical performance of a sphero-cylindrical lens. Figure 3(a) shows the propagation field generated by the horizontal and vertical axes of the lens, respectively, which coincide with the main axes of the lens. In this case, the refractive powers of each meridian are $50 \mathrm{D}$. and $33.3 \mathrm{D}$. Figures $3(b)$ and $3(c)$ represent the two Sturm [20] focals of the astigmatic lens.

Summarizing, we say that the optical performance of a lens can be studied by measurement of the propagation fields. The maximum concentration of energy in 

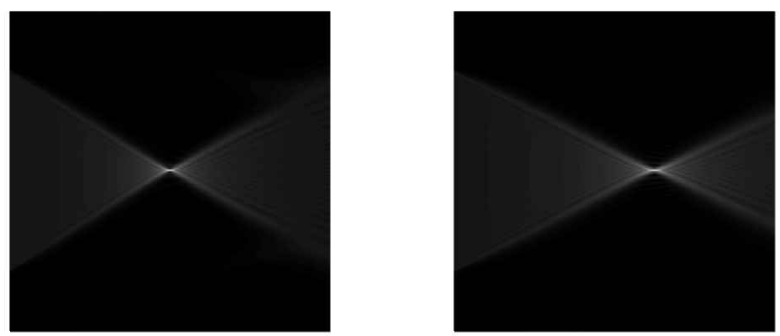

(a)
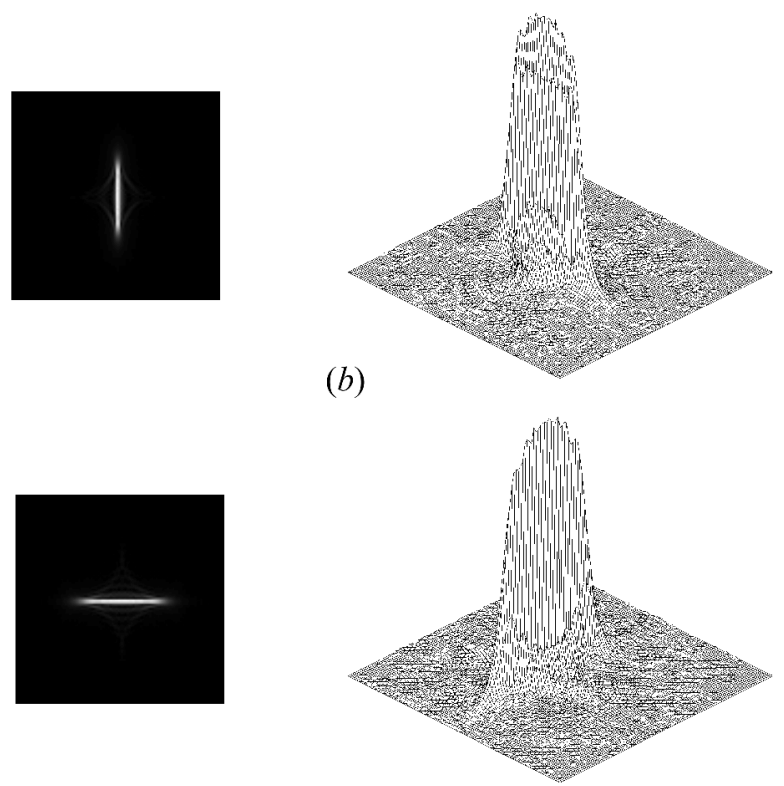

(c)

Figure 3. Sphero-cylindrical lens. (a) Propagation fields generated by the horizontal and vertical meridians respectively. (b) Light distribution at the horizontal focal plane. (c) Light distribution at the vertical focal plane.

the propagation field may be used to determine the focal length of each meridian of an arbitrary lens (i.e. the cornea). Proper choice of the principal axis of a lens will allow calculation of the principal focal lengths of that lens. From there, the bestvision point can be easily determined.

Note that any deviation from a regular surface in the lens will be observed in the propagated field and in the energy distribution at the focal plane. Hence, a complete analysis of the refracting surface can be made by studying these two plots, without a priori information of the refractive/diffractive surface. In [6] detailed studies of optical surfaces through Zernike polynomials can be found. With this technique, a priori information about the lens must be used to optimize the polynomial development. Furthermore, the computational efficiency of any operation related to the discrete Fourier transform is usually better than that obtained when dealing with a large number of polynomials of different orders. In any case, it is our feeling that dealing with the Fresnel integral and propagated 


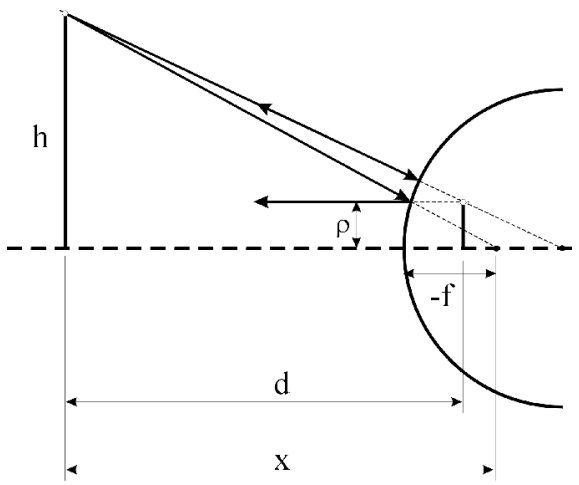

(a)

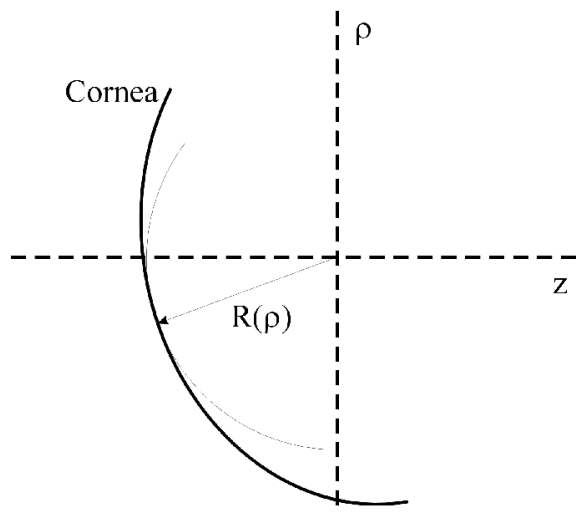

(b)

Figure 4. Principles of keratography. (a) Geometric ray-tracing of a spherical convex mirror. (b) Schematic representation of the relation between the measured radii and the corneal surface.

fields is more intuitive and exact than other methods, since we work with the surface itself, instead of an arbitrary good approximation of it.

\section{Videokeratographies and corneal height maps}

In recent years, videokeratoskopes have found widespread application in keratoconus screening, contact lens fitting and pre- and post-operative corneal refractive surgery evaluation. Most commercially available devices are based upon the Placido disc setup although alternative technologies exist. The Placido disc is a series of concentric illuminated rings which are reflected from the cornea and viewed with a video camera. The ring spacings in the resultant image are used to determine the local slope at an array of corneal points.

Geometric optics of a spherical reflecting surface are the basis for keratoscopy [5]. Let us consider one single ring from the Placido object of known radius $h$ (see figure $4(a)$ ). Ray tracing sets up similar triangles and the following relationship: 


$$
\frac{\rho}{h}=\frac{-f}{x}
$$

where $\rho$ is the linear image size, $f$ is the convex-mirror focal length and $x$ is the distance from the object to the mirror focal plane. For a spherical mirror, the focal length is related to the mirror radius of curvature through $f=R / 2$, and thus:

$$
\frac{\rho}{h}=\frac{-R}{2 x} \text {. }
$$

The distances from the Placido rings to the focal plane of the spherical convex mirror is unknown, but can be closely approximated by the distance from the rings to the image $d$. This distance is fixed by the focal length of the optical instrument that captures the final image. Finally, the radius of curvature for each point can be determined through

$$
R=\frac{-2 d_{\rho}}{h}
$$

From these data, one can easily obtain the height map of a cornea. The main assumption that has to be made here is that each corneal semi-meridian is a plane smooth curve in the sense that a curvature exists at each point [6]. In a twodimensional rectangular system $(\rho, z)$ (remember that $\rho$ is the distance of the corneal point to the optical axis and $z$ the height of the cornea) the semi-meridian with azimuth $\phi^{0}$ can be described by

$$
z=g\left(\rho, \phi^{0}\right) \text {. }
$$

As we said previously, the topography data consist of a series of radii $R\left(\rho_{i}\right)$ along each meridian at distances $\rho_{i}$ from the $z$ axis. These radii have not to be interpreted as radii of curvature. Rather, $R(\rho)$ is identified as the radius of the unique circle which is tangent to the curve at position $\rho$ and has its centre on the $z$-axis (see figure $4(b))[21,22]$. The slope of such a circle at a distance $\rho$ from the $z$ axis is $\rho /\left[R^{2}(\rho)-\rho^{2}\right]^{1 / 2}$. This quantity is, therefore, equal to the derivative of $g\left(\rho, \phi_{0}\right)$ at point $\rho$ :

$$
\frac{\partial g\left(\rho, \phi^{0}\right)}{\partial \rho}=\frac{\rho}{\sqrt{R^{2}(\rho)-\rho^{2}}}
$$

and, thus, the corneal surface can be described by:

$$
g\left(\rho, \phi^{0}\right)=\int_{0}^{\rho} \frac{\rho^{\prime}}{\sqrt{R^{2}\left(\rho^{\prime}\right)-\rho^{\prime 2}}} \mathrm{~d}_{\rho}^{\prime}
$$

The sagittal depths have thus been estimated for each meridian by numerically integrating the right side of equation (15). To do this, a value for $g\left(0, \phi_{0}\right)$ is needed. This value will be chosen equal to the average of several points around the vertex point.

In figure 5(a) we depict a standard keratograph taken with an EyeSys system. The values of the radii are colour-coded and the equivalence is provided together with the topography in the form of a colour scale.

One may ask why this kind of representation is used instead of taking a surface map by rastering the cornea, which provides directly a height map. Consider the following example: a topographic representation of the earth surface with a relief map. The equatorial radius of curvature of the earth is 3963 miles, and the peak to 
valley (under the sea) variation is approximately 12 miles. In relative terms, the variations in the elevation of continents and oceans is three orders of magnitude smaller than the radius of the earth. A relief map of the topography of the earth to scale will appear almost spherical, with elevation fluctuations of mountain ranges and ocean trenches obscured by the overall surface of the earth. This is exactly what happens with the cornea.

To reveal the fine corneal topography, a reference surface must be removed from the surface obtained. Correct choice of this reference is critical to show the small deviations from sphericity that occur in the cornea. Thus, a useful representation of the cornea globe will take the form of a residual map.

Topography maps measured through Placido disc technology provide corneal maps which can be interpreted rapidly with a very simple device, and with no need for a reference surface, this is the reason of their widespread acceptance. Notice that passing from one representation to another can be done easily with modern computers, and the final choice between one or other technology will mostly depend on the relation between price and quality.

\section{Results}

At this point, with the corneal surface obtained through videokeratography and the propagated field that it generates measured, we will be able to study the optical performance of the human cornea. In the remainder of this section several corneas will be studied. First, a brief note about the methodology employed. Note that the topographies usually are not complete due to the presence of the nose and eyelids; therefore an interpolation algorithm is needed. In order to minimize the effect of these points, only the central part of the cornea is considered here. Since the pupil of the eye in normal daylight is about $4 \mathrm{~mm}$ wide, for the corneas analysed a circular pupil of $5.5 \mathrm{~mm}$ diameter (over $8 \mathrm{~mm}$ of the entire map) is used. In cases where it becomes necessary, a simple reconstruction algorithm based on taking the average over an entire ring, will be used.

Figure 5 shows a normal cornea. This eye corresponds to a subject with no myopia or astigmatism. Figure $5(b)$ shows the propagated field generated by this cornea. This field is to be quite 'clean', similar to the one generated by the phase object depicted in figure 2 .

One important parameter used to evaluate the quality of an optical surface is the point spread function. The focal plane of a converging lens contains the image of an object located at an infinite distance in front of it. Hence, this image can be considered as the impulse response of a refractive surface for the plane located at $z=-\infty$. This parameter will be used here. The narrower the peak obtained there, the better the optical quality of the cornea. The focal plane of the cornea in figure $5(a)$ is depicted in figure 5(c). As can be observed, the peak is of very good quality (i.e. sharp and narrow), and so the cornea is also of 'good quality'.

Figure 6 shows an astigmatic and myopic cornea $(43.4 \mathrm{D}$ in the $x$-direction and $44.7 \mathrm{D}$ in the $y$-direction). Since we are comparing $43 \mathrm{D}$ resulting from the spherical curvature of the cornea with an astigmatism of less than $2 \mathrm{D}$, this is difficult to observe in the figure due to the reduced scale used. However, it can be measured directly from the numerical data. Because no irregular aberrations are present in the cornea, the propagation field for both horizontal and vertical meridians appears symmetric with respect to the $z$-axis, without any deviation 


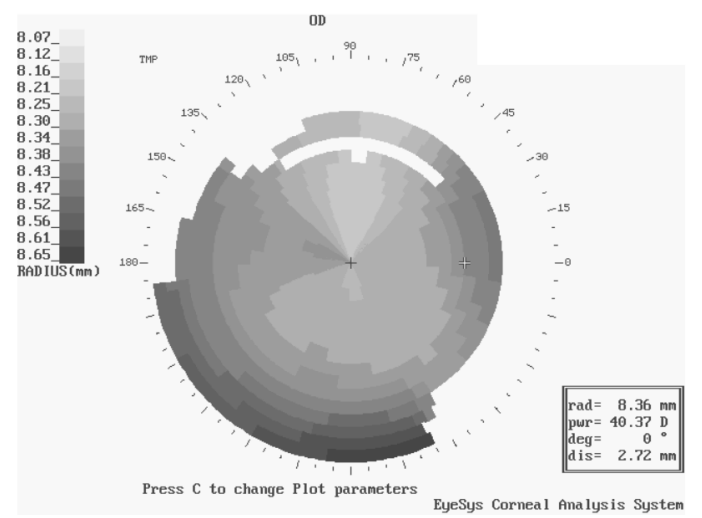

(a)
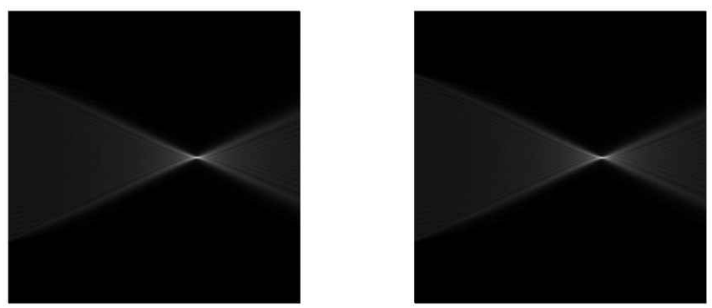

(b)
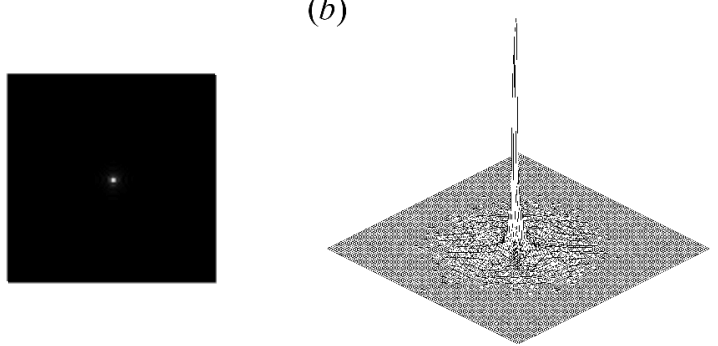

(c)

Figure 5. Normal cornea $(41.75 \mathrm{D}, 41.75 \mathrm{D})$. (a) Videokeratography; (b) propagation fields generated by the horizontal and vertical meridians respectively; $(c)$ light distribution at the focal plane.

from an ideal regular astigmatic surface (figure 3). For an astigmatic surface, two different focalization planes appear. In this case, it is more interesting to study the 'best vision point', obtained as the average of the two focus positions. This is plotted in figure 6(c). The function there is also narrow and clean, and with accurate measurements it can be shown to be slightly wider in the $y$-axis than the one in figure 5 , due to astigmatism.

A megalocornea is presented in figure 7. This pathology consists of an abnormally large cornea which, in principle, will not affect the optical quality of the surface. As can be seen in figure $7(b)$, and figure $7(c)$, the propagation fields are similar to the corresponding plots in figure $5(b)(c)$. The only remarkable fact about this cornea, beside its size, is that its refractive power is about $43.4 \mathrm{D}$ (myopic eye). 


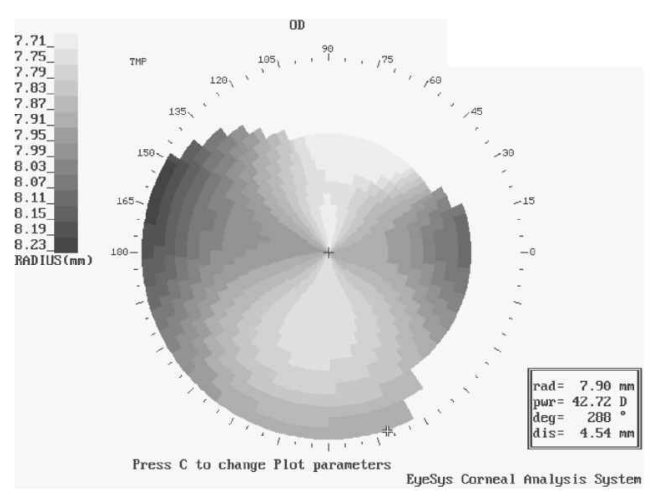

(a)
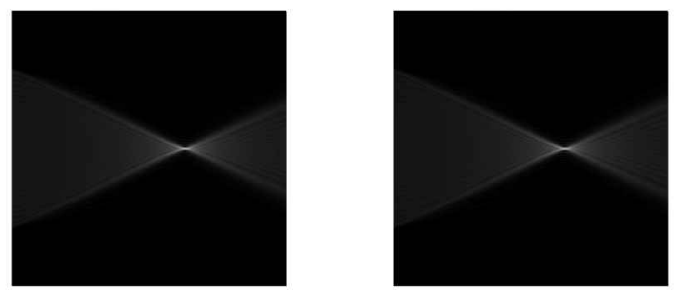

(b)
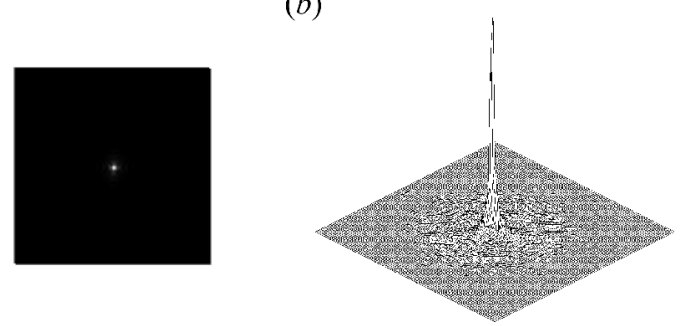

(c)

Figure 6. Astigmatic and myopic cornea (43.44 D, 44.74 D). (a) Videokeratography; (b) propagation fields generated by the horizontal and vertical meridians respectively; (c) light distribution at the 'best-vision' plane.

Figure 8 shows a cornea affected by strong astigmatism due to corneal scarring. Here, the propagated fields do not look as clean as the preceding ones. The propagation field corresponding to the horizontal meridian presents part of the energy dispersed outside the focus, and slightly directed to the bottom of the figure. This effect is more evident in the vertical-meridian propagation field. This displacement is produced by a minor curvature radius (major power) of the left and bottom part of the cornea. Hence, just by observing the propagation field we can determine if there is some irregularity in the cornea and in which direction it is located. Figure $8(c)$ shows the energy distribution at the 'best-vision' plane. It is clear that the quality of the point is poor, since it is affected by a lot of noise. In fact, when we look at the $3 \mathrm{D}$ energy distribution, we can distinguish two separated peaks. As is indicated in previous paragraphs, this is produced by a poor quality cornea. 


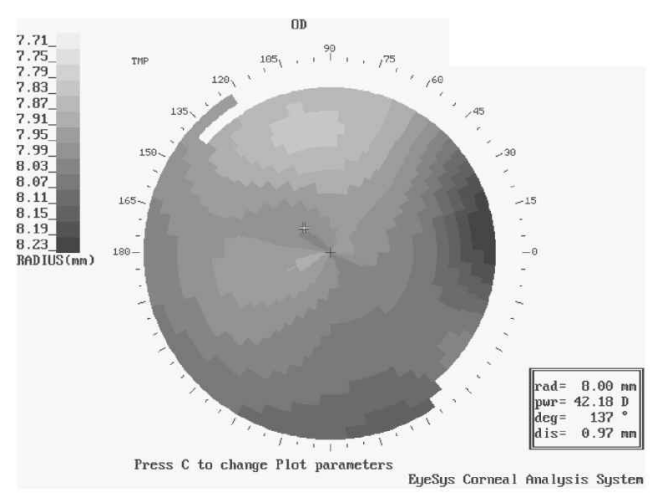

(a)
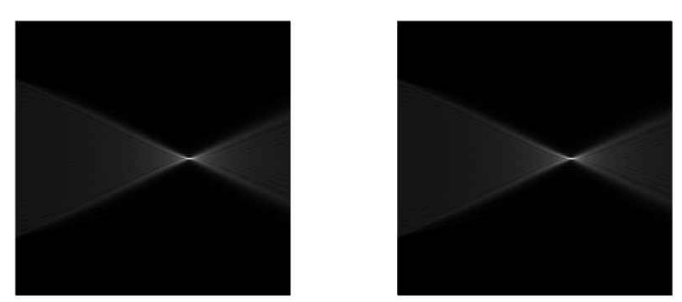

(b)
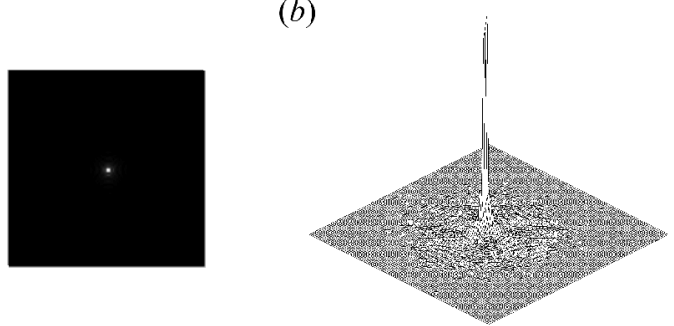

(c)

Figure 7. Megalocornea (43.44 D, 43.44 D). (a) Videokeratography; (b) propagation fields generated by the horizontal and vertical meridians respectively; $(c)$ light distribution at the focal plane.

Figures 9 to 11 show corneas affected by keratoconus in different stages of advancement. This malformation is caused by degeneration of the corneal surface. It appears like a conus coming out the cornea in the bottom part of the sphere. On the keratograph, the presence of a keratoconus can be recognized by an irregular power increase (decreasing radii) in the lower part of the image. It can also be interpreted as a strong irregular astigmatism.

The effect of a keratoconus over the propagation field is clear, since we can interpret the damaged cornea as two separated surfaces encrusted one into the other. Each of these two surfaces generates its own field, and the two overlay. As was pointed out in a previous paragraph, the focus will be displaced towards the part of the eye with the higher refractive power. This is exactly what can be observed in the propagated fields of figures 9 to 11 .

Again, the asymmetries of the diffraction fields will cause blurred optical focuses, affecting the vision quality of the subject. Notice that the form of the 


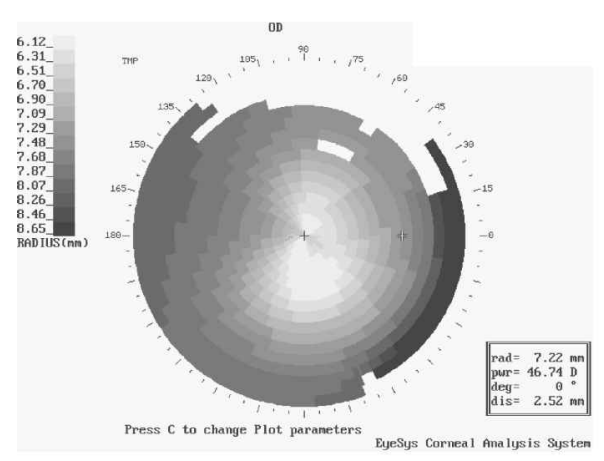

(a)
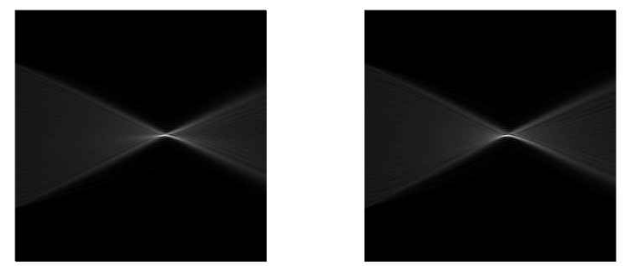

(b)
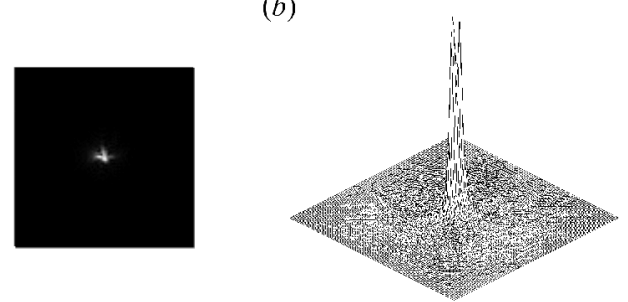

(c)

Figure 8. Damaged cornea (49.23 D, 54.04 D). (a) Videokeratography; (b) propagation fields generated by the horizontal and vertical meridians respectively; $(c)$ light distribution at the 'best-vision' plane.

diffracted fields and of the focus in all cases of keratoconus is not regular, and thus this malformation does not admit standard optical compensation, so that surgery is the only solution.

As a special case, in figure 12 the topography of a keratoconus is shown. Since the cornea presents a large number of irregularities, the keratograph cannot obtain a complete map and interpolation becomes necessary. If we apply the same methodology that was used in the previous cases, we obtain the figures $12(b)$ and 12(c). Although they seem to correspond to a keratoconus, the difference between the refractive powers obtained for the two axes $(70.9 \mathrm{D}$ and $72.9 \mathrm{D})$ is too low. Recall that a keratoconus may be identified by large irregular astigmatism, which does not correspond with the measured value.

This apparent contradiction may be caused by the interpolation algorithm. Reconstruction of a normal cornea will not present any difficulty, since the surface is regular. In contrast, when the algorithm is applied to an irregular cornea, the predictions are not so precise since the results can be distorted. This can be overcome by taking a small pupil around the corneal apex. This solution implies 


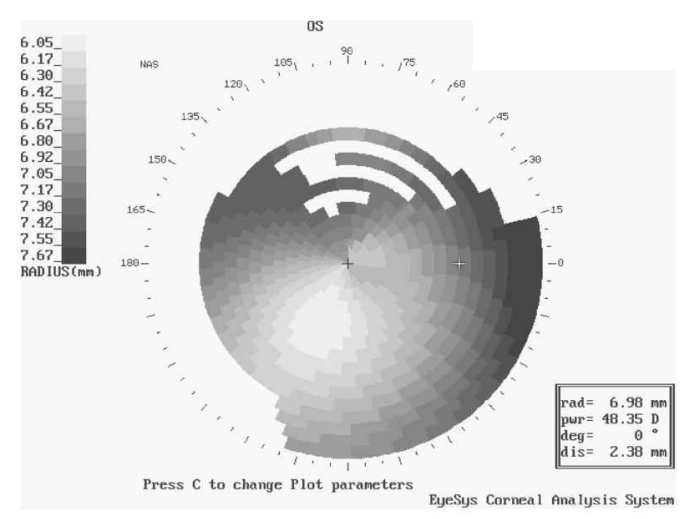

(a)
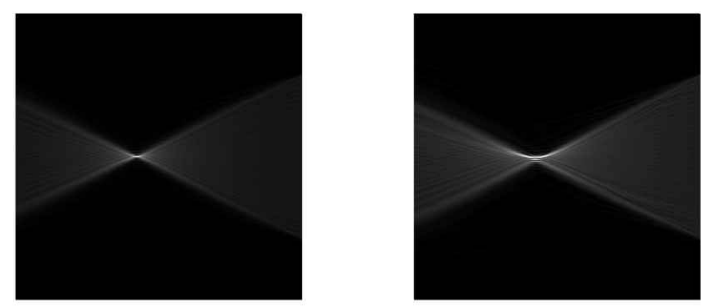

(b)
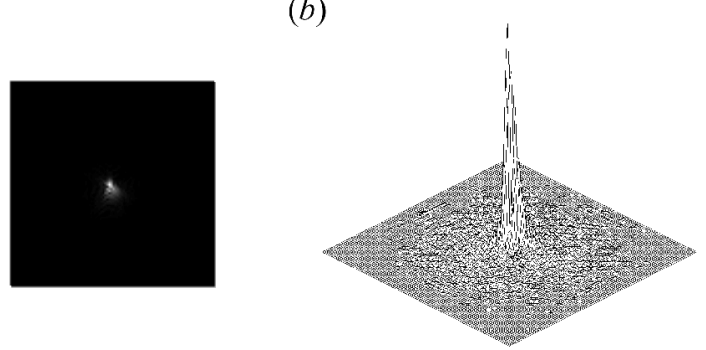

(c)

Figure 9. Cornea affected by an incipient keratoconus $(52.08 \mathrm{D}, \quad 55.55 \mathrm{D}) . \quad(a)$ Videokeratography; $(b)$ propagation fields generated by the horizontal and vertical meridians respectively; $(c)$ light distribution at the 'best-vision' plane.

data losses that may be important for the diagnosis of certain irregularities. Unfortunately, most topographies of pathologic corneas look like this last one, and the results obtained through propagation must be completed with additional information. This limitation can be overcome by the use of last generation videokeratographs.

Summarizing, we are able to clearly identify local irregularities of the cornea simply by studying the propagation fields generated by significant meridians. The method provides intuitive results with easy interpretations and provides immediate access to any propagation plane. Although coherent propagation has been used here, this study can be extended to incoherent light, thus a complete analysis of the visual performance of the eye (from an optical point of view) may be affordable. Unfortunately, as yet, the results obtained are limited by the technology that is actually available in opthalmological clinics. 


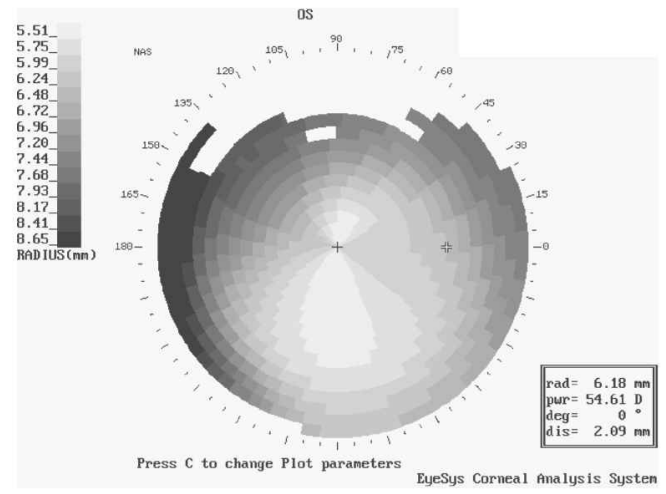

(a)
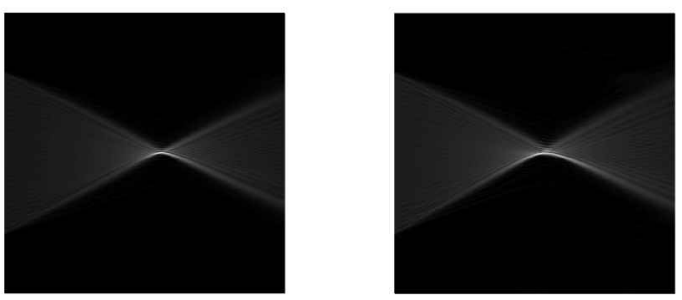

(b)
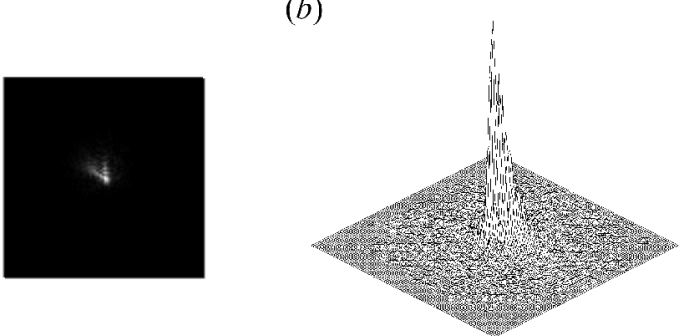

(c)

Figure 10. Cornea affected by a keratoconus (55.55 D, 61.94 D). (a) Videokeratography; (b) propagation fields generated by the horizontal and vertical meridians respectively; $(c)$ light distribution at the 'best-vision' plane.

\section{Conclusion}

This paper describes the development of a new technique to analyse the optical quality of the human cornea. Several corneas have been studied and some irregularities have been clearly recognized.

The corneal surface is directly derived from videokeratographic data. The surface obtained is then converted to a phase surface (i.e. a lens). The behaviour of a parallel light beam after passing through the lens is studied in two perpendicular meridians. In the cases described, we analyse propagation in horizontal and vertical axes.

The point of maximum energy concentration is defined as the focus of the lens. In cases where two different focuses appear for the two orthogonal meridians, the 'plane of best vision' is analysed. This point must be considered as the point spread function of the cornea corresponding to an impulse coming from infinity. The 


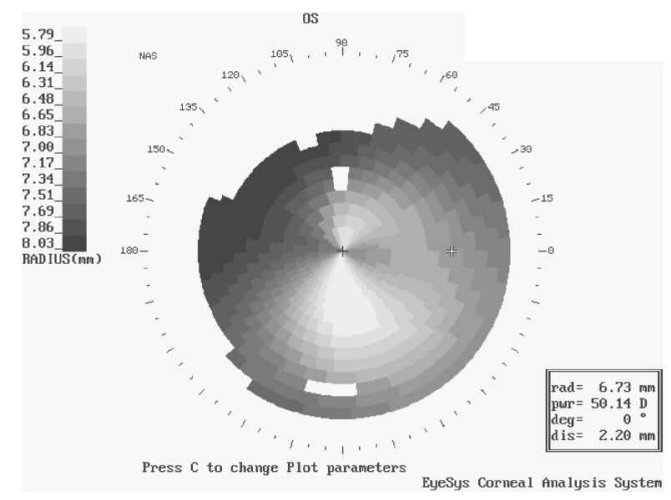

(a)
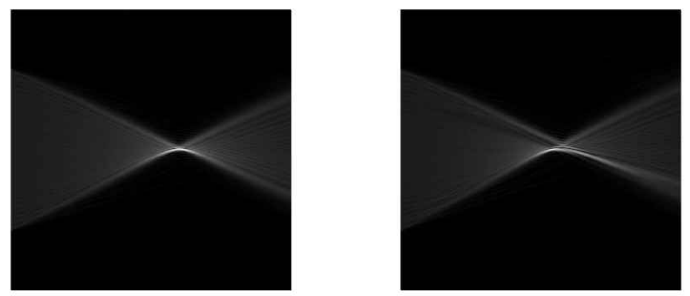

(b)
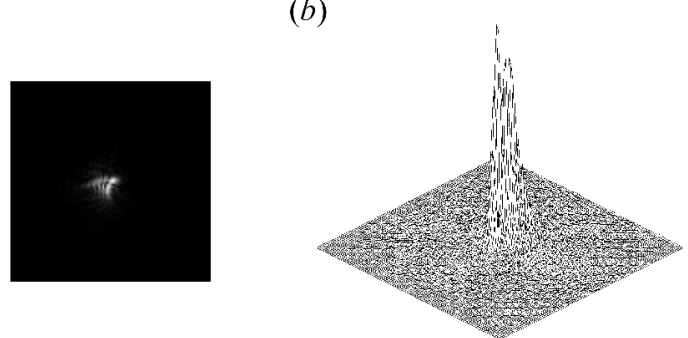

(c)

Figure 11. Cornea affected by a keratoconus (46.50 D, 58.67 D). (a) Videokeratography; (b) propagation fields generated by the horizontal and vertical meridians respectively; $(c)$ light distribution at the 'best-vision' plane.

energy distribution in this plane may be analysed in order to determine the optical quality of the cornea.

In this study the presence of keratoconus has been clearly recognized and differenced from astigmatism or myopia. Notice that here only a qualitative study of the optical performance of the cornea has been made. Exhaustive analysis of the corneal surface will require a large number of corneas and comparisons with other existing methods, and this is outside the scope of this present work.

The technique proposed may complement the surface analysis done through Zernike polynomials [6]. The cornea can first be studied through the propagation patterns it generates, and then these patterns can be explained with a Zernike development of the surface. On the other hand, the calculated surface can be studied with the help of the propagation planes, which can be compared with those obtained from the real cornea. 


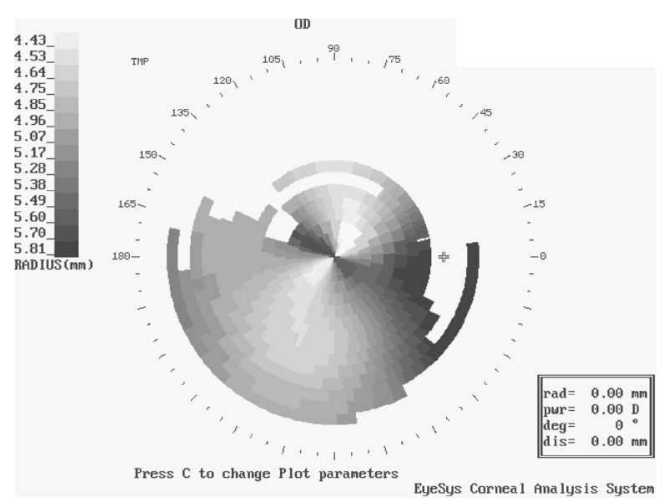

(a)
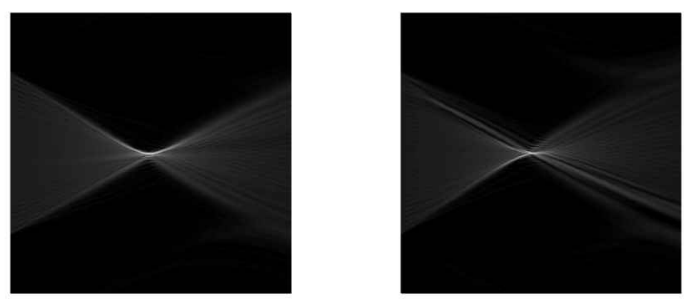

(b)
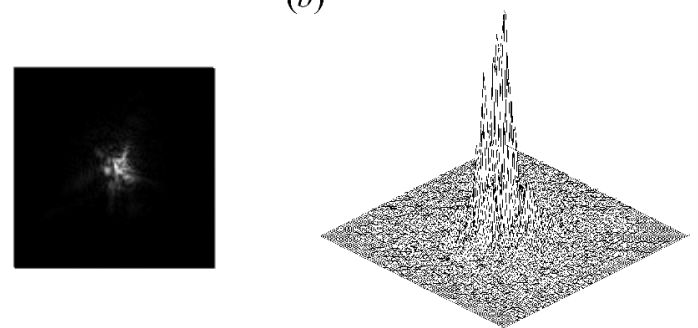

(c)

Figure 12. Cornea affected by a keratoconus (70.91 D, 72.85 D). (a) Videokeratography; (b) propagation fields generated by the horizontal and vertical meridians respectively; $(c)$ light distribution at the 'best-vision' plane.

Finally, note that this analysis can also be combined with the results obtained with the double-pass technique [1]. Once the optical performance of the cornea is substracted from that obtained for the whole eye, we will have instant access to the inner part of the eye, which cannot be accessed any other way.

\section{References}

[1] Williams, D. R., Brainard, D. H., McMahon, M. J., and Navarro, R., 1994, J. Opt. Soc. Am. A, 11, 3123.

[2] Liang, J., Grimm, B., Goelz, S., and Bille, J. F., 1994, J. Opt. Soc. Am. A, 11, 1949.

[3] Lorente, A., Artigas, J. M., Felipe, A., Pons, A. M., and Gomez J., 1999, J. Mod. Optics, 40, 1079.

[4] Jenkins, T. C. A., 1963, British J. Physiol. Optics, 20, 59. 
[5] Horner, D. G., Salmon, T. O., and Soni, P. S., 1998, Corneal topography. In Borish's Clinical Refraction, edited by W. J. Benjamin (Philadelphia: W. B. Saunders), pp. 524-558.

[6] Hemenger, R. P., Tomlinson, A., and Oliver K., 1995, Ophthalmic and Physiogical. Optics, 15, 63.

[7] Born, M., and Wolf, E., 1993, Principles of Optics (Oxford: Pergamon Press), pp. 464466.

[8] Klein, S. A., and Mandell, R. B., 1995, Investigative Ophthalmic and Visual Sci., 36, 2096.

[9] Mendlovic, D., and Ozaktas, H. M., 1993, J. Opt. Soc. Am. A, 10, 1875.

[10] Lohmann, A. W., 1993, J. Opt. Soc. Am. A, 10, 2181.

[11] Lohmann, A. W., Mendlovic, D., and Zalevsky, Z., 1998, Fractional transformations in optics. In Progress in Optics XXXVIII, edited by E. Wolf, (Elsevier Science B. V.), pp. 263-343.

[12] Alieva, T., Lopez, V., Agullo-Lopez, F., and Almeida, L. B., 1994, J. Mod. Optics, 41, 1037.

[13] Mas, D., Ferreira, C., and García, J., 1998, Fresnel diffraction calculation through the fast fractional Fourier transform, Proceedings of the SPIE 3490, 461.

[14] Mas, D., Ferreira, C., Garcia, J., and Bernardo, L. M., 2000, Opt. Eng, 39, 1427.

[15] Mas, D., Garcia, J., Ferreira, C., Bernardo, L. M., and Marinho, F., 1999, Optics Commun., 164, 233.

[16] Pons, A. M., Lorente, A., Illueca, C., Mas, D., and Artigas, J. M., 1999, J. Mod. Optics, 46, 1043.

[17] Garcia, J., Mas, D., and Dorsch, R. G., 1996, Appl. Optics, 35, 7013.

[18] Marinho, F. J., and Bernardo, L. M., 1998, J. Opt. Soc. of Am. A, 15, 2111.

[19] Gonzalez, R. C., and Witz, P. 1987, Digital Image Processing (Massachusets: Addison Wesley) pp. 100-109.

[20] Jalie, M., 1988, The Principles of Ophthalmic Lenses (London: Assoc. British Dispensing Opticians), p. 280.

[21] Applegate, R. A., and Howland, H. C., 1993, Optom. Vision Sci., 12, Suppl. 20 Abstract.

[22] Mandell, R. B., 1992, Contact Lens Association of Ophthalmol. J., 18, 267. 